Pacific Journal of Mathematics

REGULAR-CLOSED SPACES AND PROXIMITIES 


\section{REGULAR-CLOSED SPACES AND PROXIMITIES}

\section{DOUGLAS HARRIS}

The theory of the compactifications of a completely regular space has been elucidated in recent years by the theory of proximities, introduced by Efremovič and developed especially by Smirnov. The two fundamental results are that a space has a compactification if and only if it has the topology of some proximity, and that there is a one-to-one correspondence from the collection of compactifications of a space onto the collection of proximities that give the topology of the space. We shall generalize these results to a larger class of spaces, which are related to the regular-closed spaces in the same manner as completely regular spaces are related to compact spaces.

We recall that a space is said to be regular-closed if it is regular, and cannot be nontrivially densely embedded in a regular space. Since every compact space is regular-closed, then any completely regular space can be embedded in a regular-closed space, namely any compactification of it. It has been shown by Berri and Sorgenfrey [1] that a regular-closed space need not be compact, and it has been shown by Herrlich [4] that there is a regular space that cannot be densely embedded in a regular-closed space. It follows from these remarks that the class of spaces which can be densely embedded in a regularclosed space, which we call the class of $R C$-regular spaces, lies properly between the class of regular spaces and the class of completely regular spaces.

The preceding remarks lead us to ask for a characterization of those spaces that can be densely embedded in a regular-closed space. We provide such a characterization in terms of a generalization of the theory of proximities to the theory of $R C$-proximities, and we also establish a one-to-one onto correspondence between regular-closed embeddings and $R C$-proximities for an $R C$-regular space.

The term regular as used herein includes $T_{1}$ separation.

2. Proximities. We introduce axioms describing a relation between subsets of a space, which we shall call a proximity relation. These axioms are those of the usual theory of proximities, which we shall call the theory of completely regular proximities, with the exception of the axiom of complete regularity, which we do not use.

A proximity on $X$ is a symmetric relation $\delta$ between subsets of $X$ satisfying the following four conditions: 
P1. $\varnothing \phi A$ for every $A \subset X$ ( $\delta$ means "not- $\delta ")$.

P2. $A \delta A$ for every $A \neq \varnothing$.

P3. $A \delta(B \cup C)$ if and only if $A \delta B$ or $A \delta C$.

P4. If $x$ and $y$ are distinct points of $X$, then $\{x\} \phi\{y\}$.

These axioms are equivalent to those given by Katētov and Frolík in $[2,25]$. They lead to an operator $u$ on $2^{X}$ defined by $u A=\{x:\{x\} \delta A\}$. This operator $u$ has the following properties:

2.1. $u \varnothing=\varnothing$.

2.2. $A \subset u A$ for each $A \subset X$.

2.3. $u(A \cup B)=u A \cup u B$.

The operator $u$ is thus a closure operator (in the sense of $[2,25]$ ). It induces a topology whose closed sets are precisely the fixed sets under the operator $u$, that is, the sets $A$ such that $u A=A$. It need not be true that the operator $u$ is a topological closure operator, that is, we need not have $u u A=u A$ for each $A \subset X$. However, we shall shortly introduce axioms which will lead to this additional property.

We shall write $A<B$ if $A \phi X-B$, and shall say that $B$ is a proximal neighborhood of $A$. We shall also write $x \delta A$ and $x<A$ respectively in place of $\{x\} \delta A$ and $\{x\}<A$.

The relation $<$ and the operator $u$ have the following useful properties:

2.4. If $A \subset B<C<D \subset E$, then we have $A<C, C<E, A<E$, and $X-E \subset X-D<X-C<X-B \subset X-A$.

2.5. If $A<B$ and $C<D$, then $A \cap C<B \cap D$.

2.6. If $A<B$, then $A \subset u A \subset B$ and $A \subset X-u(X-B) \subset B$.

3. $R$-proximities. The next axiom will ensure that $u$ is a topological closure operator, and that the topology induced by the proximity via the closure operator $u$ is regular.

P5. (Axiom of regularity.) If $x<A$, then there is $B \subset X$ with $x<B<A$.

We define a filter to be round if for each member $V$ of the filter there is a member $W$ of the filter such that $W<V$. Axiom P5 can be restated thus: the filter of proximal neighborhoods of a point is round.

Lemma 1. A proximity on $X$ satisfying $\mathrm{P} 1-\mathrm{P} 5$ induces a closure operator $u$ such that $u u A=u A$ for each $A \subset X$. The topology induced by the proximity is regular, and $u$ is the closure operator induced by the topology.

Proof. To verify that $u u A=u A$, we need only show that 
$u u A \subset u A$. Now if $x \notin u A$, we have $x \phi A$, that is, $x<X-A$, so by P5 there is $B \subset X$ with $x<B<X-A$. Applying 2.6 we have $B \subset X-u A \subset X-A$, and hence from 2.4 we have $x<X-u A$, which is equivalent to $x \notin u u A$.

We have now shown that $u$ is the closure operator of the topology that it induces: the closed sets are precisely the sets of the form $u A$ for some $A \subset X$. Now this fact along with 2.6 shows that the proximal neighborhood filter of each point of $X$ is a regular filter (that is, a filter with a base of open sets and a base of closed sets). In particular the proximal neighborhood filter of each point is contained in the neighborhood filter of the point. Since by the definition of the topology the converse inclusion also holds we have equality of the two filters. We have therefore that the neighborhood filter of each point of the space is a regular filter, that is, the topology is regular.

An $R$-proximity is a proximity satisfying P1-P5. In accordance with Lemma 1, the topology induced by an $R$-proximity is regular. Our next result includes a generalization of the converse of Lemma 1.

Lemma 2. Suppose $Z$ is a regular topological space, and that $X$ is a dense subspace of $Z$. Define a relation between subsets of $X$ by setting $A \delta B$ if $\mathrm{cl}_{Z} A \cap \mathrm{cl}_{Z} B \neq \varnothing$.

(a) The relation $\delta$ is an $R$-proximity on $X$.

(b) A filter on $X$ is round if and only if it is the trace of a filter that is regular on $Z$.

Proof. (a) Symmetry, P1, P2, and P4 are immediate, and P3 follows from the distributivity of closure with finite union. To show $\mathrm{P} 5$, we observe that if $V$ is a neighborhood (in $X$ ) of $x \in X$, there is a closed neighborhood (in $Z$ ) $B$ of $x \in Z$ and an open neighborhood (in $Z$ ) $W$ of $x \in Z$ such that $W \cap X=V$ and $B \subset W$. Setting $A=B \cap X$ we find $x<A<V$.

(b) Suppose $\gamma$ is a round filter on $X$, and let $\zeta$ be the filter on $Z$ generated by $\left\{\mathrm{cl}_{Z} F: F \in \gamma\right\}$. Then $\zeta$ certainly has a base of closed sets. Now if $F \in \gamma$ and $G \in \gamma$ with $G<F$, we have $\operatorname{cl}_{Z} G \cap \mathrm{cl}_{Z}(X-F)=\varnothing$. Since $X$ is dense in $Z$, we also have $\operatorname{cl}_{Z} F \cup \mathrm{cl}_{Z}(X-F)=Z$. It follows that $\mathrm{cl}_{Z} G \subset Z-\mathrm{cl}_{Z}(X-F) \subset \mathrm{cl}_{Z} F$, and we have thus shown that $\zeta$ also has a base of open sets. Thus $\zeta$ is a regular filter, and it clearly induces $\gamma$ on $X$.

Conversely, suppose $\zeta$ is a regular filter on $Z$. Since $X$ is dense in $Z$ and $\zeta$ has a base of open sets, every member of $\zeta$ intersects $X$ and so the trace $\gamma$ on $X$ of $\zeta$ exists. If $V \in \gamma$, so that $V=W \cap X$ for some open set $W \in \zeta$, we let $P$ be any member of $\zeta$ such that $\operatorname{cl}_{z} P \subset W$. Then if $Q=P \cap X$ we have $\operatorname{cl}_{z} Q \cap \mathrm{cl}_{z}(X-V)=\varnothing$, so $Q<V$ and $Q \in \gamma$. Thus we have shown that $\gamma$ is round. 
The proximity defined on a regular space by declaring sets to be near if their closures intersect is, according to Lemma 2, an $R$ proximity that induces the topology of the space. We can now state the following:

THEOREM A. A topology is regular if and only if it is the topology induced by an $R$-proximity.

There may of course be many $R$-proximities that induce a given regular topology.

We now establish some properties of round filters with respect to an $R$-proximity that will be needed in the next section.

3.1. Every round filter is a regular filter.

3.2. Every neighborhood filter is maximal round.

3.3. Every round filter is contained in a maximal round filter.

3.4. Distinct maximal round filters contain disjoint open members.

Proofs. Property 3.1 follows from Lemma 1 and from 2.6. Property 3.2 follows from 3.1 along with the facts that neighborhood filters are round and maximal regular in a regular space. Property 3.3 is established in the usual manner using Zorn's lemma. To show 3.4, we observe that by 3.1 round filters are open filters. Also if the sup of two round filters is a filter, then by 2.5 , this sup is a round filter. Thus if two round filters do not contain disjoint open sets, then their sup is a round filter containing each, and this establishes 3.4 .

4. $R C$-proximities. We shall now give the axiom that is used for the connection with regular-closed spaces. It deals with a different type of neighborhood relation between subsets of an $R$-proximity space $X$.

We say that the subset $B$ of $X$ surrounds the subset $A$ if every maximal round filter that intersects $A$ (that is, every member of the filter intersects $A$ ) contains $B$.

P6. (Axiom of $R C$-regularity.) The subset $B$ surrounds the subset $A$ if and only if $B>A$.

An $R C$-proximity is a proximity that satisfies P1-P6. Since such a proximity is in particular an $R$-proximity, the induced topology is regular. We shall show in $\S 6$ that the induced topology is also $R C$ regular. The following result gives the converse, that every $R C$ - 
regular space has its topology induced by an $R C$-proximity.

LEMMA 3. Let $Z$ be a regular-closed topological space, and let $X$ be a dense subspace of $Z$. Let $\delta$ be the R-proximity induced on $X$ by $Z$ by the method described in Lemma 2.

(a) The relation $\delta$ is an $R C$-proximity on $X$.

(b) The maximal round filters on $X$ are precisely the traces on $X$ of the neighborhood filters of points of $Z$.

Proof. We show (b) first. Since $Z$ is regular, by Lemma 2(b) the trace $\gamma$ on $X$ of the neighborhood filter $\zeta$ of a point $z \in Z$ is a round filter. If $\eta$ is round and $\gamma \subset \eta$, by Lemma 2(b) there is a regular filter $\nu$ on $Z$ whose trace on $X$ is $\eta$. Since $\zeta$ is maximal regular, we must have $\nu \subset \zeta$ and thus $\eta \subset \gamma$. Conversely, if $\gamma$ is a maximal round filter it is the trace on $X$ of a regular filter on $Z$, and since $Z$ is regular-closed this regular filter has a cluster point. The trace on $X$ of the neighborhood filter of this cluster point must be the given maximal round filter.

To show (a), suppose that $A$ and $B$ are subsets of $X$ and $A>B$. By the definition of the proximity this is equivalent to $\operatorname{cl}_{Z}(X-A) \cap \operatorname{cl}_{z} B=\varnothing$. Now if $\gamma$ is a maximal round filter on $X$ then by (b) we know that $\gamma$ is the trace on $X$ of the neighborhood filter of some point $z \in Z$. If $\gamma$ intersects $B$ then $z \in \mathrm{cl}_{z} B$, and so there is a neighborhood $V$ of $z$ disjoint from $X-A$, from which we find that $A \in \gamma$. We have thus shown that if $A>B$ then $A$ surrounds $B$.

Conversely, suppose $A$ and $B$ are subsets of $X$ and $A$ surrounds $B$. Let $z \in \operatorname{cl}_{Z} B$ and let $\gamma$ be the trace of $X$ of the neighborhood filter of $z$. Then by (b) $\gamma$ is a maximal round filter. Since $\gamma$ intersects $B$ we must have $A \in \gamma$, from which it follows that $z \notin \operatorname{cl}_{z}(X-A)$. Thus $A>B$.

In accordance with this lemma, we can now state:

TheOREM B. Every RC-regular space has its topology induced by an RC-proximity.

5. Absolutely closed $R C$-proximities. We now introduce a completeness condition on $R C$-proximities that is a generalization of a condition given by Smirnov [7]. It will prove to be characteristic for regular-closed spaces in the same way that Smirnov's condition is characteristic for compact spaces. We also show that a regular-closed space has topology induced by precisely one $R C$-proximity, just as a compact space has topology induced by precisely one completely regular proximity.

An $R C$-proximity space is absolutely closed if every maximal round 
filter is the proximal neighborhood filter of some point of the space (that is, converges in the topology induced by the proximity). It will later become apparent that this is equivalent to stating that there is no proper dense embedding (in either the proximal or topological sense) of the space into an $R C$ - proximity space, which is the condition corresponding to Smirnov's definition.

THEOREM C. If an RC-proximity space is absolutely closed, then its induced topology is a regular-closed topology, and the proximity is given by: $A$ and $B$ are far if and only if they have disjoint closures.

Proof. We establish the second statement first. Suppose $A$ and $B$ are subsets of an $R C$-proximity space $X$, and that $A \varnothing B$, that is, $A<X-B$. Since the proximity satisfies $\mathrm{P} 6$ we have that $X-B$ surrounds $A$, and so every maximal round filter that intersects $A$ contains $X-B$. Now by 3.1 neighborhood filters are maximal round, and thus we see that any neighborhood filter that intersects $A$ fails to intersect $B$, that is, $A$ and $B$ have disjoint closures.

Conversely, suppose $A \delta B$, that is, $A \nless X-B$. Then by $\mathrm{P} 6$ $X-B$ does not surround $A$, so there is some maximal round filter that intersects $A$ and intersects $B$. Since we are assuming that the proximity is absolutely closed, this maximal round filter must be the neighborhood filter of some point of the space, and this point is in the closure of both $A$ and $B$.

Having characterized the proximity, we establish that the induced topology is regular-closed. According to 3.1 every round filter is a regular filter. Observing that every open set containing a closed set is a round neighborhood of the closed set, by the above characterization of the proximity, we see that every regular filter is a round filter, thus every maximal regular filter converges and the topology is regular-closed.

The following theorem is a generalization of Theorem 8 in [7].

THEOREM D. An RC-proximity space is absolutely closed if and only if the induced topology is regular closed.

Proof. That an absolutely closed $R C$-proximity induces a regularclosed topology is a part of Theorem C. To show the converse, that an $R C$-proximity space whose induced topology is regular-closed is absolutely closed, we observe that a maximal round filter (being a regular filter by 3.1) must have a cluster point, to which it must then converge (since neighborhood filters are round by 3.2). 
Using Theorems B, C, and D, we can establish the following two results.

THEOREM E. A topological space is regular-closed if and only if it has the topology induced by an absolutely closed RC-proximity.

THEOREM F. There is precisely one RC-proximity that induces the topology of a regular-closed space.

6. The ideal space of an $R C$-proximity. The final link in our chain connecting $R C$-proximities and $R C$-regular spaces is to show that a space having topology induced by an $R C$-proximity is an $R C$ regular space, and it is to this problem that we now turn our attention.

Let $\delta$ be an $R C$-proximity on $X$. We shall construct a set $r X$ and an absolutely closed $R C$-proximity $\pi$ on $r X$ such that $X$ is naturally embedded in $r X$ as a dense subspace both in the topological and the proximal sense.

Let $r X$ be the disjoint union of $X$ with an index set for the family of nonconvergent maximal round filters on $X$. For $p \in r X$, define $O^{p}$ as follows: if $p \in X$ then $O^{p}$ is the filter of proximal neighborhoods of $p$, and if $p \in r X-X$ then $O^{p}$ is the nonconvergent maximal round filter for which $p$ is the index.

Define a relation $\pi$ on subsets of $r X$ by $P \pi Q$ if there is $p \in r X$ such that for each $V \in O^{p}$ there is $(a, b) \in P \times Q$ with $V \in O^{a}$ and $V \in O^{b}$. We shall show that $\pi$ is an absolutely closed $R C$-proximity on $r X$, that it induces the proximity $\delta$ on the subset $X$, and that every point of $r X$ is related to $X$ under $\pi$. An immediate consequence will be that the topology induced on $X$ by $\delta$ is $R C$-regular.

Axioms P1 and P2 are clear, and P3 is readily shown. Axiom $\mathrm{P} 4$ follows from 3.4. Since the relation $\delta$ satisfies $\mathrm{P} 6$, it is easy to see that for subsets $A$ and $B$ of $X$, we have $A \delta B$ if and only if $A \pi B$; thus the relation $\pi$ does indeed induce the relation $\delta$ on the subset $X$. To show that every point of $r X$ is related to $X$ under $\pi$ we merely observe that if $p \in r X$ and $V \in O^{p}$, there is $x \in X$ with $V \in O^{x}$.

We now introduce some useful notation. For $A \subset X$, let $A^{\prime}=$ $\left\{p \in r X: A \in O^{p}\right\}$, and let $A^{*}=A \cup A^{\prime}$. Also let $A^{\circ}=\{x \in X: x<A\}$. It is easy to see that $\left(A^{\circ}\right)^{\prime}=\left(A^{\circ}\right)^{*}=A^{\prime}$. Given a filter $\gamma$ on $X$, we let $\gamma^{*}$ be the filter on $r X$ generated by $\left\{F^{*}: F \in \gamma\right\}$. Finally, we write $P \leqq Q$ for $P \pi(r X-Q)$.

The following lemma is useful in proving that $\pi$ has the properties P5 and P6.

LEMmA 4. (a) $p \leqq R$ if and only if there is $V \in O^{p}$ with $V^{\prime} \subset R$. 
(b) For $A, B \subset X, A^{*} \leqq B^{*}$ if and only if $A<B$.

(c) If $\zeta$ is a round filter on $r X$, then the trace $\gamma$ of $\zeta$ on $X$ exists and $\zeta=\gamma^{*}$.

(d) $\gamma^{*}$ is a (maximal) round filter on $r X$ if and only if $\gamma$ is a (maximal) round filter on $X$.

Proof. (a) If $\{p\} \pi r X-R$, then since for each $V \in O^{p}$ there is $p \in\{p\}$ with $V \in O^{p}$, we must have some $V \in O^{p}$ such that $V \notin O^{b}$ for any $b \in r X-R$; equivalently, $V^{\prime} \subset R$. Conversely, if $\{p\} \pi(r X-R)$, it is easily seen that we must have $V^{\prime} \not \subset R$ for each $V \in O^{p}$.

(b) Suppose that $A \delta(X-B)$. Then using $\mathrm{P} 6$ we see that there is $O^{p}$ such that $O^{p}$ intersects $A$ and $O^{p}$ intersects $X-B$. Since $A \subset A^{*}$ and $X-B \subset r X-B^{*}$, we have $A^{*} \pi\left(r X-B^{*}\right)$. Conversely, suppose that $A^{*} \pi\left(r X-B^{*}\right)$. Then there is $O^{p}$ such that for each $V \in O^{p}$ there is $(a, b) \in A^{*} \times\left(r X-B^{*}\right)$ with $V \in O^{a}$ and $V \in O^{b}$. Now if $a \in A$ then $a \in A \cap V \neq \varnothing$; if $a \in A^{*}-A$ then $a \in A^{\prime}$, so $A \in O^{a}$, and since also $V \in O^{a}$ we have again $A \cap V \neq \varnothing$. If $b \in\left(r X-B^{*}\right) \cap X$ then $b \in(X-B) \cap V$; if $b \in\left(r X-B^{*}\right)-X$ then $B \notin O^{b}$, thus $V \not \subset B$, and so $V \cap(X-B) \neq \varnothing$. Therefore the set $B$ does not surround $A$ and so by P6 we have $B \ngtr A$, that is, $A \delta(X-B)$.

(c) Let $A \in \zeta$; since $\zeta$ is round there is $B \in \zeta$ with $A \geqq B$. Since $B \neq \varnothing$ there is $p \in B$, and thus $p \leqq A$. It follows immediately from (a) that $A \cap X \neq \varnothing$. We have thus shown that every member of $\zeta$ intersects $X$, and so the trace filter $\gamma$ of $\zeta$ on $X$ exists.

We shall now show that $(B \cap X)^{*} \subset A$, which will establish that $\zeta \subset \gamma^{*}$. We certainly have $B \cap X \subset A \cap X$; now if $p \in(B \cap X)^{*}-(B \cap X)$, then $B \cap X \in O^{p}$, and so for each $V \in O^{p}$ there is $b \in B \cap X$ with $B \cap X \in O^{b}$, thus since $B \pi(r X-A)$ there must be $W \in O^{p}$ with $W^{\prime} \subset A$, and this gives $p \in W^{\prime} \subset A$.

To show conversely that $\gamma^{*} \subset \zeta$ we shall show that $B \subset(A \cap X)^{*}$. If $p \in B$, then $p \leqq A$, and so by using (a) we have $(A \cap X) \in O^{p}$, which gives $p \in(A \subset X)^{*}$.

(d) This follows immediately from (b) and (c).

Lemma 4 (c) and (d) establish a one-to-one correspondence from the maximal round filters on $X$ onto the maximal round filters on $r X$. They show in particular that the maximal round filters on $r X$ are precisely the filters $\left(O^{p}\right)^{*}$ for some $p \in r X$.

It is immediate from Lemma 4 (a) and (b) that $\pi$ satisfies P5. We now demonstrate that $\pi$ also satisfies $P 6$. Suppose $P, Q \subset r X$ and $P \pi Q$. Then there is a maximal round filter $0^{p}$ on $X$ such that for each $V \in O^{p}$ there is $(p, q) \in P \times Q$ with $V \in O^{p}$ and $V \in O^{q}$; then the maximal round filter $\left(O^{p}\right)^{*}$ on $r X$ intersects $P$ and does not contain 
$r X-Q$, so $r X-Q$ does not surround $P$.

Conversely, suppose $r X-Q$ does not surround $P$. Then there is a maximal round filter on $r X$ that intersects $P$ and does not contain $r X-Q$, that is, it intersects $P$ and $Q$. Letting $O^{p}$ be the trace of this filter on $X$, we have that for each $V \in O^{p}$ there is $(a, b) \in P \times Q$ such that $V \in O^{a}$ and $V \in O^{b}$, and therefore $P \pi Q$.

We have now shown that $\pi$ satisfies $\mathrm{P} 1-\mathrm{P} 6$, that is, that $\pi$ is an $R C$-proximity on $r X$, and we have shown that $\pi$ induces $\delta$ on its subspace $X$, and that $X$ is proximally dense in $r X$. This establishes in particular that the space $X$ with the topology induced by $\delta$ is densely embedded in the space $r X$ with the topology induced by $\pi$. If we can show that $r X$ with this topology is regular-closed, then we will have shown that $X$ with topology induced by $\delta$ is $R C$-regular. To show that $r X$ is regular-closed, we show that $\pi$ is absolutely closed and apply Theorem $\mathrm{C}$.

The proximity $\pi$ is absolutely closed if every maximal round filter on $r X$ converges in the topology of the proximity. Now one need only observe that a maximal round filter on $r X$ is of the form $\left(O^{p}\right)^{*}$ for some $p \in r X$, and that the following sets are all bases for $\left(O^{p}\right)^{*}:\left\{V^{*} V \in O^{p}\right\} ;\left\{V^{\prime}: V \in O^{p}\right\}$; and $\{A \subset r X: p \leqq A\}$. Thus, $\left(O^{p}\right)^{*}$ converges to $p \in r X$.

The set $r X$ with the proximity $\pi$ is called the ideal space of the proximity $\delta$. Summing up the preceding conclusions, we have the following result:

Theorem G. The ideal space of an $R C$-proximity is an absolutely closed RC-proximity space, and its induced topology is regular-closed. The given space is a dense subspace of its ideal space, in both the topological and the proximal sense.

COROLlary. Every RC-proximity space is RC-regular.

The following theorem, which is a generalization of the corresponding result for completely regular proximities, is an immediate consequence of the preceding results.

THEOREM H. There is a one-to-one correspondence from the collection of $R C$-proximities for an $R C$-regular space onto the collection of regular-closed embeddings of the space, given by letting an $R C$ proximity correspond to its ideal space.

We order regular-closed embeddings of an $R C$-regular space by stating $Z>Y$ if there is a map $h$ from the regular-closed embedding $Z$ (necessarily) onto the regular-closed embedding $Y$ that reduces to 
the identity on the subspace $X$. Then it is not difficult to show that the corresponding proximities are comparable, in the sense that sets which are far in the proximity of $Y$ are far in the proximity of $Z$ when we have $Z>Y$. The author does not know if the converse holds, that is, if comparable proximities give rise to comparable regular-closed embeddings.

7. Some problems and remarks. There are many unsettled questions in the theory of regular-closed spaces, and it is hoped that the preceding theory will be helpful in their solution. It allows us to focus attention on the space itself, rather than on its extensions, and it gives us a method of comparing different regular-closed embeddings by their effect on the space.

Problem I. This problem has already been mentioned. It is to determine if comparable proximities give rise to comparable regularclosed embeddings.

Problem II. In the theory of compactifications it is known how to determine if a map from a completely regular space $X$ has an extension to a given compactification of $X$. Similar results have been established for Hausdorff-closed spaces [3], [4], [5], [6]. It would be quite useful to have such a theory for maps from an $R C$-regular space into a regular-closed space.

Problem III. In analogy with the theory of compactifications, and with the theory of Hausdorff-closed spaces, we might conjecture that there is a largest regular-closed embedding for each $R C$-regular space $X$.

Problem IV. It is unknown whether the product of $R C$-regular spaces is $R C$-regular, and whether the product of regular-closed spaces is regular-closed. A related question is whether the product of $R C$ proximities (using the definition of product given in [2, 39]) is an $R C$-proximity.

Problem V. There is no nontrivial characterization of the regularclosed subspaces of a regular-closed space.

Professor Mark Mandelker is due special thanks for his encouragement and advice. Additional thanks are due the referee for his detailed criticism and advice. 


\section{REFERENCES}

1. M. P. Berri and R. H. Sorgenfrey, Minimal regular spaces, Proc. Amer. Math. Soc. 14 (1963), 454-458.

2. E. Cech, Topological spaces, Interscience, London, 1966.

3. D. Harris, Structures in topology, (to appear)

4. H. Herrlich, T $T_{\nu}$-Abgeschlossenkeit und T $T_{\nu}$-Minimalität, Math. Z. 88 (1965), 285-294.

5. C. T. Liu, Absolutely closed spaces, Trans. Amer. Math. Soc. 130 (1968), 86-104.

6. J. Porter and J. Thomas, On H-closed and minimal Hausdorff spaces, Trans. Amer. Math. Soc. 138 (1969), 159-170.

7. Ju. M. Smirnov, On proximity spaces, Mat. Sb. (N.S.) 31 (1952), 543-574; Amer. Math. Soc. Trans. (2) 38 (1964), 5-36.

Received June 9, 1969, and in revised form January 16, 1970. The author is grateful to the University of Kansas for a Graduate Council Honors Fellowship that has supported this work.

UNIVERSITY OF KANSAS

LAWRENCE, Kansas

Marquette University

Milwaukee, Wisconsin 



\section{PACIFIC JOURNAL OF MATHEMATICS}

\section{EDITORS}

H. SAMELSON

Stanford University

Stanford, California 94305

\section{Richard Pierce}

University of Washington

Seattle, Washington 98105
J. DugundJI

Department of Mathematics

University of Southern California

Los Angeles, California 90007

RICHARD ARENS

University of California

Los Angeles, California 90024

\section{ASSOCIATE EDITORS}

\section{E. F. BeCKenBACH}

B. H. NeUmanN
K. YosHida

\section{SUPPORTING INSTITUTIONS}

\author{
UNIVERSITY OF BRITISH COLUMBIA \\ CALIFORNIA INSTITUTE OF TECHNOLOGY \\ UNIVERSITY OF CALIFORNIA \\ MONTANA STATE UNIVERSITY \\ UNIVERSITY OF NEVADA \\ NEW MEXICO STATE UNIVERSITY \\ OREGON STATE UNIVERSITY \\ UNIVERSITY OF OREGON \\ OSAKA UNIVERSITY \\ UNIVERSITY OF SOUTHERN CALIFORNIA
}

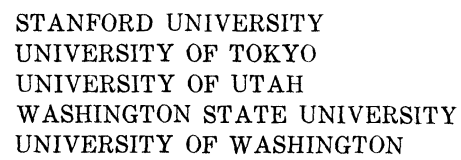

STANFORD UNIVERSITY UNIVERSITY OF TOKYO

UNIVERSITY OF UTAH

WASHINGTON STATE UNIVERSITY

UNIVERSITY OF WASHINGTON

The Supporting Institutions listed above contribute to the cost of publication of this Journal, but they are not owners or publishers and have no responsibility for its content or policies.

Mathematical papers intended for publication in the Pacific Journal of Mathematics should be in typed form or offset-reproduced, (not dittoed), double spaced with large margins. Underline Greek letters in red, German in green, and script in blue. The first paragraph or two must be capable of being used separately as a synopsis of the entire paper. The editorial "we" must not be used in the synopsis, and items of the bibliography should not be cited there unless absolutely necessary, in which case they must be identified by author and Journal, rather than by item number. Manuscripts, in duplicate if possible, may be sent to any one of the four editors. Please classify according to the scheme of Math. Rev. Index to Vol. 39. All other communications to the editors should be addressed to the managing editor, Richard Arens, University of California, Los Angeles, California, 90024.

50 reprints are provided free for each article; additional copies may be obtained at cost in multiples of 50 .

The Pacific Journal of Mathematics is published monthly. Effective with Volume 16 the price per volume (3 numbers) is $\$ 8.00$; single issues, $\$ 3.00$. Special price for current issues to individual faculty members of supporting institutions and to individual members of the American Mathematical Society: $\$ 4.00$ per volume; single issues $\$ 1.50$. Back numbers are available.

Subscriptions, orders for back numbers, and changes of address should be sent to Pacific Journal of Mathematics, 103 Highland Boulevard, Berkeley, California, 94708.

PUBLISHED BY PACIFIC JOURNAL OF MATHEMATICS, A NON-PROFIT CORPORATION

Printed at Kokusai Bunken Insatsusha (International Academic Printing Co., Ltd.), 7-17, Fujimi 2-chome, Chiyoda-ku, Tokyo, Japan. 


\section{Pacific Journal of Mathematics}

\section{Vol. 34, No. 3 \\ July, 1970}

Richard Hindman Bouldin, The peturbation of the singular spectrum

Hugh D. Brunk and Søren Glud Johansen, A generalized Radon-Nikodym derivative .

Henry Werner Davis, F. J. Murray and J. K. Weber, Families of $L_{p}$-spaces

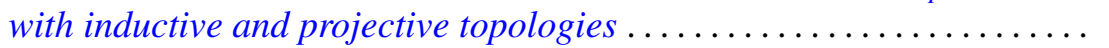

Esmond Ernest Devun, Special semigroups on the two-cell .

Murray Eisenberg and James Howard Hedlund, Expansive automorphisms

of Banach spaces ......................................

Frances F. Gulick, Actions of functions in Banach algebras.

Douglas Harris, Regular-closed spaces and proximities.

Norman Lloyd Johnson, Derivable semi-translation planes . .

Donald E. Knuth, Permutations, matrices, and generalized Young

tableaux..........................................

Herbert Frederick Kreimer, Jr., On the Galois theory of separable

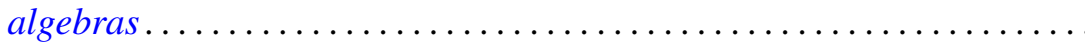

You-Feng Lin and David Alon Rose, Ascoli's theorem for spaces of

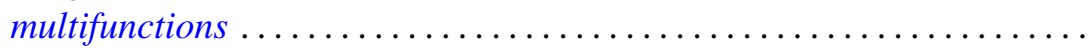

David London, Rearrangement inequalities involving convex functions . . . .

Louis Pigno, A multiplier theorem.

749

Helga Schirmer, Coincidences and fixed points of multifunctions into trees.

755

Richard A. Scoville, Some measure algebras on the integers .

Ralph Edwin Showalter, Local regularity of solutions of Sobolev-Galpern

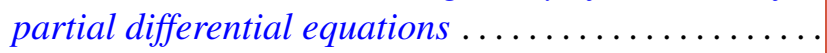

Allan John Sieradski, Twisted self-homotopy equivalences

John H. Smith, On S-units almost generated by S-units of subfields ...

803

Masamichi Takesaki, Algebraic equivalence of locally normal

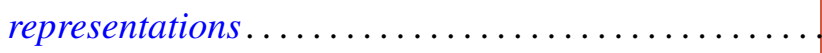

Joseph Earl Valentine, An analogue of Ptolemy's theorem and its converse in

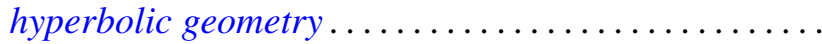

David Lawrence Winter, Solvability of certain p-solvable linear groups of finite order 